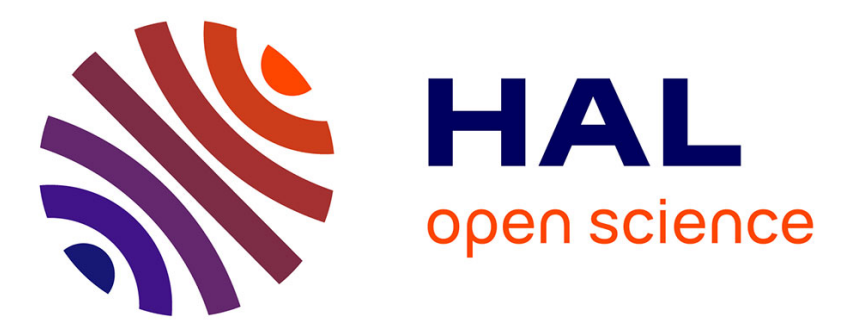

\title{
La Mise en scène du mariage dans la comédie de la Restauration: vide rituel et anti-rites
}

\author{
Florence March
}

\section{To cite this version:}

Florence March. La Mise en scène du mariage dans la comédie de la Restauration: vide rituel et anti-rites. XVII-XVIII Revue de la Société d'études anglo-américaines des XVIIe et XVIIIe siècles , 2006, Âges de la vie et rites de passage, 62, pp.51-66. halshs-01391929

\section{HAL Id: halshs-01391929 \\ https://shs.hal.science/halshs-01391929}

Submitted on 4 Nov 2016

HAL is a multi-disciplinary open access archive for the deposit and dissemination of scientific research documents, whether they are published or not. The documents may come from teaching and research institutions in France or abroad, or from public or private research centers.
L'archive ouverte pluridisciplinaire HAL, est destinée au dépôt et à la diffusion de documents scientifiques de niveau recherche, publiés ou non, émanant des établissements d'enseignement et de recherche français ou étrangers, des laboratoires publics ou privés. 


\title{
La Mise en scène du mariage dans la comédie de la Restauration : vide rituel et anti-rites
}

Florence March

\begin{abstract}
Although Restoration comedies assert the existence and the necessity of the institution of marriage, they nevertheless question some of its fundamentals. The staging of nuptial rites in particular crystallizes social and dramatic tensions without resolving them. The following study derives from the observation of two recurrent phenomena : on the one hand, ritual non-fulfilment, which results from the open-endedness of the texts and questions the performative character of the promise of marriage ; on the other hand, anti-rites or parodic substitutes for the wedding ceremony, such as dances celebrating the couple's separation, mock-marriages, and subverted proviso scenes.
\end{abstract}

\section{Résumé}

La comédie de la Restauration réaffirme l'existence et la nécessité de l'institution du mariage, tout en remettant paradoxalement en cause ses modalités. La représentation du rituel nuptial cristallise les tensions sociales et dramatiques sans les résoudre. On constate un vide rituel lié à la non-clôture du texte, qui remet en question le caractère performatif de la promesse de mariage, et la mise en scène de substituts parodiques que l'on peut qualifier d'anti-rites. Nombre de pièces procèdent à une déconstruction du rituel nuptial qui se traduit par la représentation éclatée d'une série d'antirites tels que la danse de la désunion, les mariages de dupes, les proviso scenes et leur envers.

\section{Citer ce document / Cite this document :}

March Florence. La Mise en scène du mariage dans la comédie de la Restauration : vide rituel et anti-rites . In: XVIIXVIII. Revue de la société d'études anglo-américaines des XVIle et XVIIle siècles. N62, 2006. Âges de la vie et rites de passage [Colloque tenu en Sorbonne les 25 et 26 novembre 2005] pp. 51-66;

http://www.persee.fr/doc/xvii_0291-3798_2006_num_62_1_2410

Document généré le 24/05/2016 


\title{
LA MISE EN SCÈNE DU MARIAGE DANS LA COMÉDIE DE LA RESTAURATION : \\ VIDE RITUEL ET ANTI-RITES
}

\begin{abstract}
Résumé : La comédie de la Restauration réaffirme l'existence et la nécessité de l'institution du mariage, tout en remettant paradoxalement en cause ses modalités. La représentation du rituel nuptial cristallise les tensions sociales et dramatiques sans les résoudre. On constate un vide rituel lié à la nonclôture du texte, qui remet en question le caractère performatif de la promesse de mariage, et la mise en scène de substituts parodiques que l'on peut qualifier d'anti-rites. Nombre de pièces procèdent à une déconstruction du rituel nuptial qui se traduit par la représentation éclatée d'une série d'antirites tels que la danse de la désunion, les mariages de dupes, les proviso scenes et leur envers.
\end{abstract}

\begin{abstract}
Although Restoration comedies assert the existence and the necessity of the institution of marriage, they nevertheless question some of its fundamentals. The staging of nuptial rites in particular crystallizes social and dramatic tensions without resolving them. The following study derives from the observation of two recurrent phenomena : on the one hand, ritual non-fulfilment, which results from the open-endedness of the texts and questions the performative character of the promise of marriage; on the other hand, anti-rites or parodic substitutes for the wedding ceremony, such as dances celebrating the couple's separation, mock-marriages, and subverted proviso scenes.
\end{abstract}

« Good marriages are made in heaven ». Ce proverbe anglais des XVIe et XVIIe siècles (Cressy 252) résume la vision du mariage qui prévaut dans la comédie de la Restauration : un constat pessimiste, néanmoins source d'effets comiques et de divertissement. Les dramaturges de l'époque puisent dans un fonds commun de métaphores stéréotypées qui associent invariablement le mariage à l'ennui, l'enfermement, la privation de liberté et l'échec. Cette représentation verbale du mariage se double de la mise en scène tout aussi négative d'un univers dramatique peuplé de cocus et de femmes 
trompées. Pour autant, nombre de comédies s'achèvent sur une promesse de mariage. Comment interpréter ce hiatus entre les niveaux thématique et structurel? Quel sens donner à ce parcours dramatique a priori paradoxal, condensé dans le titre d'une comédie de Durfey, The Marriage-Hater Match'd (1692)?

Pour tenter d'apporter quelques éléments de réponse, je me pencherai sur la question de la représentation du rituel nuptial, qui cristallise les tensions sociales et dramatiques. Le lecteur-spectateur fait immédiatement deux constats : d'une part, un vide rituel lié à la non-clôture du texte, qui remet en question le caractère performatif de la promesse de mariage ; d'autre part, la mise en scène de substituts parodiques que l'on peut qualifier d'anti-rites.

La présente étude s'appuie sur un corpus non exhaustif de quinze comédies qui jalonnent toute la période comprise entre 1660 et 1710 et dramatisent la problématique de la relation entre les sexes, qu'il s'agisse de crise conjugale ou des rites de séduction qui conduisent les jeunes amants au mariage. ${ }^{1}$ Certes, la comédie du mariage, et plus particulièrement la mise en scène de problèmes conjugaux, existe depuis l'Antiquité et n'a pas manqué d'être exploitée par les dramaturges anglais de la Renaissance. ${ }^{2}$ La spécificité du genre à la Restauration semble tenir à deux phénomènes nouveaux. En premier lieu, la comédie du mariage représente la majeure part de la production dramatique de l'époque. Tous les grands dramaturges et la plupart de ceux qui sont moins connus ont écrit au moins une comédie dont l'intrigue principale ou secondaire est centrée sur un conflit conjugal. Il suffit de passer en revue les titres des comédies mises en scène durant la seconde moitié du XVIIe siècle pour se convaincre que le champ sémantique du mariage domine d'un bout à l'autre de la période. Ensuite, le ton change : le scepticisme devient la

1. Le corpus se compose ainsi : Secret Love; or, The Maiden Queen (Dryden, 1667); Marriage à-la-Mode (Dryden, 1672); Epsom-Wells (Shadwell, 1672); The Country Wife (Wycherley, 1675); The Man of Mode; or, Sir Fopling Flutter (Etherege, 1676) ; A True Widow (Shadwell, 1678-79) ; The Feign'd Curtezans, or, A Night's Intrigue (Behn, 1679) ; The Souldiers' Fortune (Otway, 1680) ; The Atheist ; or, The Second Part of The Souldiers' Fortune (Otway, 1682); Sir Anthony Love; or, The Rambling Lady (Thomas Southerne, 1690); The Wives' Excuse; or, Cuckolds Make Themselves (Southerne, 1691); Love's Last Shift; or, The Fool in Fashion (Cibber, 1696); The Relapse ; or, Virtue in Danger (Vanbrugh, 1696); The Way of the World (Congreve, 1700); The Beaux' Stratagem (Farquhar, 1707).

2. Voir, par exemple, The Taming of the Shrew (1593-94) de Shakespeare. 
règle, rompant définitivement avec l'esprit de la comédie romantique qui précède. Pour tenter de rendre compte de la variété et de la complexité des cas de figure, la sélection opérée suit donc un double critère, diachronique et synchronique.

Avant d'entamer l'analyse, il convient encore de souligner qu'il existe, en matière de rite de mariage, de nombreuses divergences entre codes sociaux et dramatiques. La comédie de la Restauration présente dans ce domaine deux particularités étroitement liées, qui rendent caduque toute tentative pour y voir une projection de la réalité : d'une part, la dramatisation du mariage s'avère très peu réaliste, contrairement à la tendance esthétique qui caractérise la comédie de mœurs de l'époque $;^{3} \mathrm{~d}$ 'autre part, sur la question précise du mariage, la comédie se montre en avance sur son temps, les dramaturges ayant recours à des schémas structurels conventionnels pour donner forme aux aspirations sociales de leurs contemporains.

$\mathrm{Si}$ les comédies de la Restauration s'achèvent quasi systématiquement sur une promesse de mariage, la cérémonie rituelle n'est jamais mise en scène. Je reviendrai ultérieurement sur le cas particulier des parodies de cérémonie et de la mise en scène des précontrats qui semblent davantage s'apparenter à des anti-rites qu'à des rites. Alors que le mariage se situe au cœur du dialogue et motive l'action dramatique la plupart du temps, le rite nuptial se trouve décentré, repoussé en marge de la représentation, décalé dans un espace-temps hors-scène. Les proviso scenes ou contrats de futuro ont également pour effet de différer au-delà du tomber du rideau la cérémonie nuptiale à laquelle, d'une certaine façon, ils se substituent.

Non seulement il n'y a pas de cérémonie pour confirmer la promesse de mariage et figer l'intrigue, mais surtout ce vide rituel est associé à tout un éventail de stratégies qui sapent la clôture du texte. Des menaces pèsent sur l'avenir du jeune couple, laissant à penser qu'il pourrait peut-être renoncer à concrétiser son projet d'union horsscène. Ainsi, lorsque le rideau tombe sur The Man of Mode (1676), le mariage de Harriet et Dorimant est encore soumis à condition : le rake doit se rendre à la campagne pour y courtiser la jeune fille. L'intrigue comique du Marriage à-la-Mode (1672) de Dryden

3. Voir Alleman; pour Pearson, c'est dans le domaine du mariage que la comédie de la Restauration s'éloigne le plus de tout espèce de réalisme social (76). 
s'achève sur la réconciliation des deux couples, l'un marié, l'autre sur le point de l'être. L'on pourrait croire au rétablissement de l'ordre moral et social si Doralice n'ajoutait à leur pacte un article qui inscrit l'infidélité dans la relation conjugale : "I will add but one proviso, that whoever breaks the league, either by war abroad, or neglect at home, both the women shall revenge themselves by the help of the other party » $(4: 5.1,335)$.

Une autre stratégie consiste à mettre en scène, en contrepoint de l'intrigue centrée sur les jeunes amants, l'échec d'un couple marié. Cette projection en creux de l'avenir des futurs époux, qui dramatise le conflit entre l'amour et le mariage, fait du rituel un seuil pervers. Dès lors, le décalage de la cérémonie dans un espace-temps horsscène traduit la réticence du jeune couple à passer de l'autre côté du miroir. Epsom-Wells (1672) de Shadwell illustre cette perversion structurelle à l'extrême. La dernière scène concentre, dans un jeu parodique sur le chiffre deux qui dénote le couple, deux adultères et deux séparations de biens et de corps qui remettent en question les deux mariages prévus. L'une des jeunes filles, Lucia, souligne le lien de cause à effet : "See what Matrimony comes to-» $(2: 5.1,180)$.

Ce procédé de dédoublement en creux trouve un prolongement dans les diptyques. La double intrigue de The Souldiers' Fortune (1680) s'achève simultanément sur une promesse d'union entre Sylvia et Courtine et l'adultère de Lady Dunce. Deux ans plus tard, Otway revisite son texte pour donner une suite au dénouement laissé en suspens. The Atheist (1682) met en scène l'échec du mariage de Sylvia et Courtine qui se substituent ainsi aux Dunce, disparus de l'intrigue. À la charnière des deux pièces, la cérémonie nuptiale célébrée hors-scène apparaît vide de sens. Vanbrugh, quant à lui, réécrit dans The Relapse (1696) le dénouement de Love's Last Shift (1696) de Cibber, qui célèbre la réforme peu plausible de l'époux volage. Cette «culture de l'emprunt» (Venet 1 : clxxxi) se manifeste à plus grande échelle à travers les variations dramatiques d'un même canevas répétitif, inlassablement remis sur le métier, qui font de la comédie de la Restauration le lieu d'un «immense palimpseste » (Lagae 442) et dont la fin reste, encore et toujours, à écrire.

Ainsi, dans la comédie de la Restauration, la promesse de mariage donne l'illusion de la clôture du texte. Cette convention dramatique ne se substitue pas, dans les pièces comiques de l'époque, au rituel du 
mariage. Certes, la cérémonie nuptiale n'est guère plus représentée dans les comédies antérieures. ${ }^{4}$ Mais il semble que la promesse d'union ait alors un caractère performatif qui lui fait défaut dans la comédie de la fin du XVIIe siècle, de sorte qu'on ne peut parler à leur encontre de vide rituel. ${ }^{5}$ La structure ouverte de la comédie de la Restauration, au sens où l'entend Umberto Eco, tend à démontrer que la mise en scène de la satire du mariage n'a pas seulement fonction d'exutoire des tensions sociales. Elle ne peut se concevoir comme une parenthèse carnavalesque, un processus contrôlé célébrant le retour à l'ordre établi, puisque le dénouement reflète le scepticisme qui imprègne le texte. Les stratégies qui parasitent la promesse de mariage et sapent la clôture du texte confèrent au vide rituel, à des degrés différents selon le contexte social et les dramaturges, une dimension subversive.

Le dénouement des comédies de la Restauration est donc lourd de significations tant sur le plan dramatique que social. Dès lors que le rite de mariage est une manifestation de cohésion sociale en effet, le vide rituel signale un certain malaise. On peut y voir un reflet oblique de la confusion juridique qui caractérise l'institution du mariage de la Restauration en 1660 jusqu'au Marriage Act de 1753. Les mariages clandestins se multiplient de façon spectaculaire durant cette période. ${ }^{6}$ Les contrats verbaux, difficiles à prouver, relèvent directement du débat parlementaire sur le serment. ${ }^{7} \grave{A}$ cela, il faut ajouter la validation rétroactive des mariages civils célébrés pendant l'interrègne, et le décalage entre la réalité juridique et la tradition populaire selon laquelle des concubins, par exemple, sont considérés comme mariés. ${ }^{8}$

4. Le dénouement de As You Like It fait figure d'exception, encore que la tonalité parodique sous-jacente en fasse un cas limite. Dans Much Ado About Nothing, la cérémonie religieuse est interrompue et reportée hors-scène. Entre-temps, toutefois, les complications de l'intrigue ont été résolues et le spectateur n'a aucun doute sur l'accomplissement du rite nuptial après le tomber du rideau. Peut-être les consentements ne sont-ils jamais échangés sur scène pour éviter que les acteurs ne soient liés par un contrat de praesenti, une forme de mariage certes irrégulière mais néanmoins valide avant la loi de Hardwicke sur le mariage.

5. Les derniers vers de As You Like It soulignent la continuité entre la représentation scénique et le hors-scène : « Proceed, proceed. We'll begin these rites / As we do trust they'll end, in true delights » (5.4.195-96).

6. Stone, Uncertain Unions 22-30.

7. Voir Staves 191-251. Le chapitre 4 « Oaths and vows » est consacré au débat social et littéraire sur la question du serment.

8. Stone, Uncertain Unions 19-20 et 16. 
Protéiforme, le mariage s'avère relativement facile à contracter. Il est en revanche beaucoup plus difficile d'y mettre un terme, problème d'autant plus aigu à une époque où les historiens notent une recrudescence des mariages d'argent. ${ }^{9}$ Entre 1660 et 1753, plusieurs dizaines de projets de loi sur le mariage sont déposés pour tenter de réformer l'institution. ${ }^{10}$ Dans ce contexte, le vide rituel qui caractérise la comédie peut se lire comme la métaphore in absentia des vides juridiques et autres défauts de la législation sur le mariage. Cette stratégie d'évitement ne remet pas tant en cause l'institution que ses manques et invite le public à inscrire dans le dénouement paradoxalement inachevé les réformes auxquelles il aspire. La même démarche procède de la représentation d'anti-rites.

Nombre de pièces procèdent à une déconstruction du rituel nuptial qui se traduit par la représentation éclatée d'une série d'antirites. Je me pencherai successivement sur la danse de la désunion, les mariages de dupes, les proviso scenes et leur envers.

La comédie s'achève conventionnellement par une danse, élément constitutif du rituel nuptial et pourtant dissocié de lui dans les pièces étudiées. D'une part, la danse intervient avant la cérémonie religieuse censée se dérouler hors-scène, selon un processus d'inversion qui perturbe la logique du rituel. D'autre part, on l'a vu précédemment, rien n'est moins sûr que l'accomplissement des autres rites. Ce morcellement du rituel va à l'encontre de sa fonction de cohésion sociale. À cela s'ajoute la mise en œuvre d'autres stratégies qui pervertissent le sens de la danse en désolidarisant les niveaux structurel et sémantique.

Le dénouement de The Country Wife (1675) de Wycherley met en scène une farandole de cocus. Celle-ci conclut dans un même mouvement, sous le signe du scepticisme, l'action principale centrée sur Horner, dont le nom évoque la fonction dramatique, et l'action secondaire qui s'achève sur une promesse de mariage entre Alithea et Harcourt. Le commentaire de Pinchwife qui précède immédiatement la danse souligne sans ambiguïté sa fonction de trait d'union satirique

9. La commercialisation du mariage entre 1650 et 1750 s'expliquerait par la révision de la loi sur la propriété terrienne qui renforce le droit d'aînesse (Simmons 25-48). Voir aussi Stone, Uncertain Unions 83-104.

10. Simmons 40. 
entre les deux intrigues : "Cuckolds like Lovers shou'd themselves deceive » (5.4.411). Le rite demeure festif mais se teinte ouvertement de cynisme puisque le verbe «deceive» présente d'emblée la cohésion sociale comme une illusion. La danse renforce au plan visuel la fonction de sape déjà remplie au niveau verbal par les divers commentaires qui fusent à l'annonce du mariage d'Alithea et Harcourt :

$\begin{array}{ll}\text { ALITHEA } & \begin{array}{l}\text { There's doctrine for all Husbands Mr. Harcourt. } \\ \text { HARCOURT }\end{array} \\ \text { I edifie Madam so much, that I am impatient till I } \\ \text { am one. } \\ \text { DORILANT } & \begin{array}{l}\text { And I edifie so much by example I will never be } \\ \text { one. }\end{array} \\ \text { SPARKISH } & \text { And because I will not disparage my parts I'le } \\ & \text { ne're be one. } \\ \text { HORNER } & \begin{array}{l}\text { And I alass can't be one. } \\ \text { PINCHWIFE }\end{array} \\ & \begin{array}{l}\text { But I must be one- against my will } \\ (5.4 .387-9 .\end{array}\end{array}$

Ce détournement du rite de la danse, dont l'harmonie gestuelle collective consiste théoriquement en une variation métaphorique sur le serment prononcé par les nouveaux époux et leur union physique, trouve un écho dans la fin du dialogue. Le passage de la prose aux vers confère au texte une certaine solennité, encore accentuée par l'emploi du distique dont les vers rimant deux à deux évoquent l'union conjugale. Toutefois, cet échange rimé a lieu entre le cocu et le rake Horner dont les paroles respectivement désabusées et cyniques consacrent la rupture du fond et de la forme, de la structure et du sens. L'infidélité, sinon la séparation, semblent d'ores et déjà inscrits dans le rituel nuptial.

Shadwell exploite dans Epsom-Wells une variante du procédé. Puisque la pièce ne s'achève sur aucune promesse de mariage, Woodly propose de ne pas déroger à la convention dramatique de la danse finale en célébrant sa rupture avec sa femme : "To shew you, that there was never yet so decent a Divorce, I have Fiddles to play at it, as they use to do at Weddings » $(2: 5.1,181)$. Dès lors qu'il n'y a pas d'adéquation entre la gestuelle et le sens dont elle est rituellement 
investie, cette danse de la rupture apparaît comme une coquille vide, un signifiant à la recherche de son signifié. ${ }^{11}$

La comédie de la Restauration regorge de parodies de mariage, dans lesquelles l'un des futurs époux ignore la véritable identité de l'autre, masqué ou déguisé, et le célébrant lui-même se révèle bien souvent être un imposteur. Elle place donc le mariage sous le signe de la méprise et ce, dès la célébration du rituel. En désignant presque systématiquement le mari comme bouc émissaire, la comédie prend une revanche sur le réel. ${ }^{12}$

Dans The Country Wife, Pinchwife a arrangé le mariage de sa sœur Alithea avec Sparkish. Le jour fixé pour leur union, le fop se rend chez sa promise en compagnie d'un prêtre, qui n'est autre que son rival Harcourt en soutane. La scène qui réunit les futurs conjoints en habit de noces et le pseudo-célébrant évoque une répétition de la cérémonie religieuse qui a lieu hors-scène, autorisant ainsi le spectateur à jeter un œil en coulisse. Le faste déployé ne parvient pas à masquer l'absence de sentiments dans ce qui n'est qu'une transaction financière. Cette fêlure entre le visible et l'invisible, les codes structurels et le sens qu'ils véhiculent, favorise le développement de l'ironie dramatique. Profitant de la bêtise de Sparkish, Harcourt préserve les apparences du rituel nuptial qu'il transforme en scène de séduction. Il joue sur la polysémie des mots, sur l'ambivalence de la syntaxe et du contexte énonciatif, pour déclarer sa flamme à Alithea tout en feignant de servir les intérêts de Sparkish : «I desire nothing more than to marry you presently» (4.1.142). La déconstruction

11. Il existait différentes façons de mettre un terme à la vie de couple: la séparation à l'amiable, la séparation de corps et de biens prononcée par un tribunal ecclésiastique, l'annulation par un tribunal ecclésiastique, le divorce parlementaire à partir de 1697 (solution coûteuse, scandaleuse, qui ne concerne que trois à quatre cas par an et n'est accordée qu'aux hommes jusqu'au début du XIXe siècle). Voir Stone, Broken Lives. Des comédies de la fin de la période font allusion au divorce parlementaire, par exemple The Way of The World (1700) de Congreve (5.1), mais seules la séparation et l'annulation sont mises en scène. Voir Alleman 106-41 et Simmons 317-80.

12. Sir Anthony Love; or, The Rambling Lady (1690) de Thomas Southerne fournit un contre-exemple. La comédie met en scène deux mariages de dupe : l'union finale de Lucy avec un bouc émissaire masculin, Sir Gentle Golding, et le mariage de Violante et Sir Anthony (en réalité Lucy travestie) célébré par Ilford, déguisé en prêtre. Dans ce dernier cas, Sir Anthony cède sa place à Ilford pour consommer l'union après la cérémonie. Individualiste, l'héroïne travestie défend son propre droit à la liberté et non celui de la femme en tant que groupe social. 
parodique de l'office religieux à laquelle il se livre ne laisse pas d'évoquer le débat contemporain sur la dimension non sacramentelle $\mathrm{du}$ mariage, alimenté par l'expérience du mariage civil durant l'interrègne puritain et, par analogie, la question alors brûlante de la nature des relations entre le monarque et ses sujets ${ }^{13}$ :

ALITHEA

HARCOURT

ALITHEA

SPARKISH
Well, most reverend Doctor, pray let us make an end of this fooling.

With all my soul, Divine, Heavenly Creature, when you please.

He speaks like a Chaplain indeed.

Why, was there not, soul, Divine, Heavenly, in what he said. (4.1.123-29)

En jouant sur la fracture entre le visible et l'invisible, Harcourt procède à une défamiliarisation du rite dont la fonction de cohésion sociale provient, entre autres, de ce qu'il s'appuie sur des pratiques collectives réglées, connues et reconnues de tous. Sparkish devient sans s'en rendre compte étranger à son propre mariage, alors même qu'il est familier des conventions et met un point d'honneur à les respecter (il tient à se marier pendant les heures canoniques : " my Mother charg'd me never to be married out of the Canonical hours » (4.1.169-70); il se réfère à l'usage : " when I get me a Wife, I must get her a Chaplain, according to the Custom» (4.1.82-83, mes italiques). En revanche, pour Alithea dont le nom signifie " vérité ", cette scène initie une quête sémantique qui s'achève au tomber du rideau, lorsqu'elle fait coïncider le sens du mariage avec son amour pour Harcourt.

Un autre effet pervers de la mise en scène des mariages de dupes consiste à s'attaquer à la fonction de seuil du rituel, qui marque le

13. Staves 115-16. Selon Staves, la question de la nature non sacramentelle du mariage n'est toujours pas résolue à l'époque de la Restauration. L'article 25 des Trente-neuf Articles de l'Église d'Angleterre publiés en 1571 déclare certes que le mariage n'est pas un sacrement, mais cette affirmation qui n'a aucun caractère légal met du temps à s'imposer dans les mentalités. Il semble que la Glorieuse Révolution, qui institutionnalise une relation contractuelle et non plus divine entre le monarque et ses sujets, marque un tournant décisif dans le débat. Bishop Gilbert Burnet s'y réfère explicitement pour déclarer que le mariage n'est plus un sacrement, dix ans plus tard, dans An Exposition of the Thirty-nine Articles of the Church of England (London, 1699). Pour Burnet, le caractère prétendument indissoluble du mariage se fonde sur la croyance collective erronée que le mariage est de nature sacramentelle et non contractuelle. 
passage d'un état à un autre. Dans la dernière scène de Sir Anthony Love (1690), Lucy se fait passer pour Floriante afin d'épouser sir Gentle Golding, à qui elle a naguère été vendue par sa tante et dont elle a juré de se venger. Lorsqu'il découvre sa méprise, sir Gentle n'a d'autre alternative que la séparation immédiate, en échange d'une rente annuelle dûment négociée qui assure désormais à Lucy son indépendance financière. Le nom de sir Gentle Golding prend tout son sens dans l'accomplissement de la fonction dramatique de pourvoyeur de fonds qui y est inscrite. La fin du rituel nuptial célébré hors-scène n'est donc pas le mariage mais son envers, la séparation de biens et de corps. Contrat de mariage et contrat de rupture se télescopent dans la même séquence, bouleversant une fois de plus le contrat de spectacle, puisque la convention dramatique de l'union finale, loin de réconcilier les contraires, scelle définitivement leur antagonisme. Le mariage se trouve réduit à la seule cérémonie religieuse qui ne marque plus le passage de l'état de célibataire à celui d'époux et se trouve ainsi vidée de son sens, à un seuil absurde ouvrant sur le néant. La subversion du rituel nuptial en anti-rite sert, dans le théâtre militant de Southerne, la satire des mariages d'argent dont la femme est communément victime dans la société comme dans la comédie.

Shadwell avait déjà exploité ce procédé dix-huit ans auparavant dans la dernière scène d'Epsom-Wells. Abusé par les beaux discours de Mrs Jilt, dont le patronyme indique pourtant que le mariage sera éphémère, Clodpate l'épouse avant de réclamer la séparation séance tenante lorsqu'il découvre à qui il a véritablement affaire. Mrs Jilt constitue à ses dépens la dot qui lui manquait pour convoler en justes noces avec un autre. Elle révèle ensuite que le mariage, célébré par le domestique de Woodly déguisé en ecclésiastique, était de toute façon invalide. ${ }^{14}$ Rituel et institution, mariage et rupture sont englobés dans une vaste farce, frappés du signe de l'illusion. La séquence duplique, dans ce raccourci burlesque, l'action principale qui s'achève sur la séparation des Woodly. Dès lors, on comprend que les célibataires, en position de spectateurs dans la scène comme le souligne Lucia («See what Matrimony comes to-» $[2: 5.1,180]$, c'est moi qui souligne),

14. La comédie invalide systématiquement tout mariage célébré par un imposteur, contrairement à l'usage réel. Aussi curieux que cela puisse paraître, en effet, pareil simulacre passait pour irrégulier mais néanmoins légal au regard du droit canonique. Voir Alleman 60-79. 
refusent de faire semblant de croire à l'illusion conjugale représentée sous leurs yeux. Dans un sursaut critique, ils s'affranchissent simultanément du pacte de spectacle et du contrat de mariage: "Madam, since we cannot agree upon better tearms, let me claim your Promise, and admit me for your Servant » $(2: 5.1,181)$. Suivant l'exemple de Mrs Jilt, dont les noces de dupe s'apparentent à un brouillon rituel avant le mariage légitime, les jeunes gens affirment leur droit à l'essai : «I do receive you upon tryal » $(2: 5.1,181)$.

Les célèbres proviso scenes, encore appelées contrats de futuro ou pré-contrats de mariage, constituent un autre type de brouillon rituel. Elles ne sont pas suivies de la consommation charnelle avant le tomber du rideau, car l'héroïne doit, selon le code dramatique, satisfaire à l'idéal de virginité. Leur pouvoir performatif est donc limité, soumis à condition. Là encore, dans ce type de scène qui se substitue à la cérémonie nuptiale, les époux potentiels manifestent leur résistance à la clôture du texte.

Ils négocient pied à pied les termes d'un accord qui définit les règles de vie commune tout en préservant les individualités. Leur pacte vise à faire du mariage une union des cœurs, des corps et des volontés. Par là même, ils défient le système patriarcal selon lequel l'homme et la femme deviennent une seule personne, celle du mari dont la femme prend d'ailleurs le nom. Les futurs époux tentent donc d'établir l'équilibre et la stabilité de leurs relations, comme pour conjurer l'effet pervers du rituel nuptial. En effet, selon Isabella, personnage de la comédie A True Widow (1678) de Shadwell, la cérémonie de mariage marque le point de bascule à partir duquel les forces en présence s'inversent sans jamais s'équilibrer :

THEODOSIA

ISABELLA
[...] but I had reduced him to offer Marriage.

Then, it seems, he is weary of being your Slave, and would make you his. ${ }^{15}$ (3: 5.1, 283-363)

Dès lors, la proviso scene constitue une tentative oxymorique, d'avance vouée à l'échec, pour concilier les statuts incompatibles de célibataire et d'époux qui s'y télescopent. C'est ce qu'exprime l'engagement solennel de Cornelia dans The Feign'd Curtezans (1679) de Behn : "I do here promise to be the most Mistriss-like wife » (6: 5.1.708-9), ou la déclaration de Florimel à Celadon dans

15. La pièce fut représentée en 1678 et publiée en 1679. 
Secret Love (1667) de Dryden: «we will be married by the more agreeable names of mistress and gallant» (2: 5.1). À l'image de la cérémonie nuptiale à laquelle il se substitue, le rite de la proviso scene cristallise les tensions et devient anti-rite.

Le diptyque de Thomas Otway est un constat d'échec: le précontrat conclu entre Silvia et Courtine dans The Souldiers' Fortune ne résout en rien la dualité du code social. Après leur mariage, dans The Atheist, Courtine continue à appeler Silvia «child», terme qui dénote sa position d'autorité vis-à-vis de sa femme, qu'il juge immature. En outre, fidélité et infidélité conjugales sont simultanément inscrites dans le pré-contrat, justifiant par avance le comportement libertin de Courtine dans The Atheist. Sur ce point, le pré-contrat contredit le serment de fidélité que prêtent les époux lors de la cérémonie de mariage dont il sape a priori la signification et la valeur. Ainsi, la tentative de défier le système patriarcal aboutit paradoxalement à le réaffirmer. Le tyran domestique qu'Isabella voit en tout mari prend les traits de Mirabell dans la fameuse proviso scene de The Way of the World (1700), comme Nadia Rigaud l'a montré. En parfait diplomate, il donne à Millamant l'illusion d'une négociation dans une scène qui confirme, s'il en est besoin, la rupture $\mathrm{du}$ fond et de la forme. Le rite, sinon social du moins dramatique, de la proviso scene échoue à redonner du sens au consentement mutuel «I do ». L'union contractuelle reste un leurre dans la réalité comme dans la comédie. ${ }^{16}$

Après la Glorieuse Révolution, alors que des personnages féminins clament leur droit à la rébellion domestique, ${ }^{17}$ la relation contractuelle

16. Sur la question du contrat de mariage et de son articulation avec le contrat social dans le débat qui oppose Locke à Filmer, voir Carole Pateman et Mary Lyndon Shanley.

17. Par exemple Lady Brute: "The Argument's good between the King and the People, why not between the Husband and the Wife» (Vanbrugh, The Provok'd Wife, 1: 1.1, 116) et Mrs Sullen : "A Man dare not play the Tyrant in London, because there are so many Examples to encourage the Subject to rebel »; "But in England, a Country whose Women are it's Glory, must Women be abus'd, where Women rule, must Women be enslav'd? " (Farquhar, The Beaux' Stratagem, 2 : 2.1.121-22 et 4.1.2-5). Quelques années avant la Glorieuse Révolution, Porcia avait déjà fait le lien entre la situation politique nationale et l'ordre établi dans le foyer : « With hopes of Liberty I am indeed : It is an English Woman's natural Right. Do not our Fathers, Brothers and Kinsmen often, upon pretence of it, bid fair for Rebellion against their Sovereign? And why ought not we, by their Example, to rebel as 
basée sur le consentement mutuel est représentée avec ironie dans les scènes de séparation à l'amiable qui parodient les proviso scenes. Lorsque Mr Woodly annonce publiquement leur séparation dans la dernière scène d'Epsom-Wells, Mrs Woodly remarque : "This I think is the first time we e'er agreed since our Wedding » $(2: 5.1,180)$. Les scènes de rupture empruntent le cadre juridique du pré-contrat et la structure rituelle de la cérémonie de mariage, mais ils véhiculent un sens diamétralement opposé. ${ }^{18} \mathrm{La}$ négociation devant témoins concerne à présent les conditions de la séparation. Un à un, les différents rites sont pris à rebours: l'union des mains ou «handfasting» qui cimente rituellement l'engagement: «These Hands join'd us, these shall part us» (The Beaux' Stratagem 5.4.249); le baiser: "Faith take a Kiss at parting for old acquaintance » (Epsom-Wells, vol. $2: 5.1,180)$; la danse finale. La substitution d'une cérémonie de rupture à la convention dramatique du mariage passait encore pour une exception en 1682, comme le souligne l'épilogue d'Epsom-Wells :

\section{A Play without a Wedding, made in spight \\ Of old Black-Fryars ; 'tis a fine way they write}

Mais à la fin de la période de la Restauration, la séparation de biens et de corps est devenue convention dramatique dans les comédies. C'est ce que laisse penser le commentaire blasé du comte Bellair sur la séparation des Sullen à la fin de The Beaux' Stratagem : «Begar the Ceremony be vera pretty!» (5.4.254), même si l'accent étranger du personnage français souligne encore le processus de défamiliarisation à l'œuvre dans la parodie. Pourtant, le rite de la séparation de biens et de corps reste un leurre, un anti-rite. La femme demeure financièrement dépendante de son ex-mari et tous deux sont dans l'impossibilité de se remarier. Mrs Friendall, dans The Wives' Excuse (1692), ne cache pas son amertume : «I must be still your Wife, and still unhappy » (1: 5.3.326). Ironiquement, l'anti-rite finit

plausibly against them ?» (Otway, The Atheist or, The Second Part of The Souldiers' Fortune, 3: 5.1, 156.

18. The Man of Mode contient une parodie de pré-contrat qui scelle une rupture d'un autre type (3.1). Afin de gagner du temps, Young Bellair et Harriet miment le jeu de la séduction devant leurs parents, qui projettent de les marier. La rhétorique gestuelle est en contradiction totale avec leur pacte verbal de ne pas se marier. 
par s'ériger en rituel dramatique et s'avère tout aussi contraignant que le rite du mariage conventionnel auquel il se substitue.

Ainsi le rituel du mariage apparaît comme le lieu de tensions sociales qui ne sont pas résolues dans la comédie. Celle-ci réaffirme l'existence et la nécessité de l'institution du mariage, tout en remettant paradoxalement en cause ses modalités. Si le dénouement des pièces étudiées donne l'illusion de respecter la convention dramatique d'un point de vue structurel, il ne sanctionne pas le retour à l'ordre établi puisque le sens est constamment différé, toujours en fuite. ${ }^{19}$ Le conflit entre les sexes persiste à bas bruit dans un espacetemps hors-scène dont les contours sont toutefois esquissés, dans un entre-deux où se rejoignent peut-être fiction et réalité. La déconstruction satirique du mariage a certes pour effet d'exorciser les tensions sociales, mais le processus ne va pas à son terme et la catharsis reste inachevée (si tant est que jamais elle soit achevée). Refusant de cantonner le débat à la seule représentation, vide rituel et anti-rites ont au contraire un effet de relance, qui induit la perméabilité des espaces dramatique et réel et rend le contrat de spectacle d'autant plus complexe.

\author{
Florence MARCH \\ Université d'Avignon et des Pays du Vaucluse
}

\title{
OUVRAGES CITÉS
}

Alleman, Gellert Spenser. Matrimonial Law and the Materials of Restoration Comedy. Philadelphia, PA : U of Pennsylvania P, 1942.

BEHN, Aphra. The Feign'd Curtezans, or A Night's Intrigue. 1679. The Works of Aphra Behn. Ed. Janet Todd. Vol. 6. London: William Pickering, 1992-96. 7 vols.

19. La différance (derridienne) du sens est parfois encore accentuée par la mise en espace du texte. Le dernier acte de The Wives' Excuse, qui met en scène un adultère, puis un divorce, donne lieu à une succession de discovery scenes. Les panneaux de décor s'ouvrent à plusieurs reprises, repoussant toujours plus loin les limites contraignantes de l'espace scénique, comme si le sens du texte refusait d'y rester cantonné. 
CORDNER, Michael, ed. Four Restoration Marriage Plays. Oxford: Oxford UP, 1995.

CRESSY, David. Birth, Marriage and Death. Ritual, Religion, and the Life-Cycle in Tudor and Stuart England. Oxford: Oxford UP, 1997.

DÉPRATS, Jean-Michel et Gisèle VENET, éds. Shakespeare. Tragédies. Vol. 1. «Bibliothèque de la Pléiade ». Paris : Gallimard, 2002.

DRYDEN, John. Secret Love, or the Maiden Queen. 1667. of The Works of John Dryden. Ed. Walter Scott. Vol. 2. London, 1808. 18 vols. Project Gutenberg. April 24, $2004<$ http :// www.gutenberg.org/ files/12166/12166-8.txt>.

. Marriage à-la-Mode. 1672. The Works of John Dryden. Ed. Walter Scott. Vol. 4. London, 1808. 18 vols. Project Gutenberg. March 13, $2005<$ http ://www.gutenberg.org/files/15349/15349h/15349-h.htm>.

ECO, Umberto. L'Euvre ouverte. 1962. Trad. Chantal de Roux de Bézieux. Paris : Seuil, 1965.

FARQUHAR, George. The Beaux' Stratagem. 1707. The Works of George Farquhar. Ed. Shirley Strum Kenny. Vol. 2. Oxford: Clarendon, 1988. 2 vols.

LAGAE, Denis. "Circularités dans la comédie de la Restauration (1660-1700) ». Thèse de doctorat Université de Paris IV, 2001.

OTway, Thomas. The Atheist or, The Second Part of The Souldiers' Fortune. 1682. The Complete Works of Thomas Otway. Ed. Montague Summers. Vol. 3. Bloomsbury : Nonesuch, 1926 ; New York : AMS, 1967. 3 vols.

Pateman, Carole. The Sexual Contract. Stanford, CA : Stanford UP, 1988.

PEARSON, Jacqueline. The Prostituted Muse: Images of Women Dramatists 1642-1737. New York : Harvester, 1988.

Rigaud, Nadia J. « Millamant dans la scène du contrat de The Way of the World: un exemple de pseudo-rébellion ». Rebelles dans le monde anglo-américain aux XVIIe et XVIIIe siècles. Actes du colloque la Société d'Études Anglo-Américaines des XVIIe et XVIIIe siècles, 23 et 24 octobre 1987. Paris : Publications de l'Université de Paris III, 1989. 179-91.

SHADWELl, Thomas. Epsom-Wells. 1672. The Complete Works of Thomas Shadwell. Ed. Montague Summers. Vol. 2. London: Fortune, 1927 ; New York : Benjamin Blom, 1968. 5 vols. 
. A True Widow. 1679. The Complete Works of Thomas Shadwell. Ed. Montague Summers. Vol. 3. London: Fortune, 1927 ; New York : Benjamin Blom, 1968. 5 vols.

SHAKESPEARE, William. As You Like It. 1599-1600. The Complete Works. Ed. Stanley Wells and Gary Taylor. Oxford : Clarendon, 1988.

SHANLEY, Mary Lyndon. « Marriage Contract and Social Contract in Seventeenth-Century English Political Thought ». Western Political Quarterly 32 : 1 (1979): 79-91.

SimMONS, Eva. "'Virtue Intire' : Aphra Behn's Contribution, in her Comedies, to the Marriage Debates of the Seventeenth Century ». Diss. University of London, 1990.

SOUTHERnE, Thomas. The Wives' Excuse; or, Cuckolds Make Themselves. 1692. The Works of Thomas Southerne. Ed. Robert Jordan and Harold Love. Vol. 1. Oxford : Clarendon, 1988. 2 vols.

Staves, Susan. Players' Scepters: Fictions of Authority in the Restoration. Lincoln : U of Nebraska P, 1979.

STONE, Lawrence. Uncertain Unions. Marriage in England 1660-1753. Oxford : Oxford UP, 1992.

. Broken Lives. Separation and Divorce in England 1660-1857. Oxford : Oxford UP, 1993.

VAnBrugh, John. The Provok'd Wife. 1697. The Works of Sir John Vanbrugh. Ed. Bonamy Dobrée and Geoffrey Webb. Vol. 1. Bloomsbury : Nonesuch, 1927 ; New York : AMS, 1967. 4 vols. WYCHERLEY, William. The Country Wife. 1675. The Plays of William Wycherley. Ed. Arthur Friedman. Oxford : Clarendon, 1979. 